\title{
Cochrane in CORR
}

\section{Cochrane in CORR ${ }^{\mathbb{R}}$ : Manual Therapy and Exercise for Rotator Cuff Disease}

\author{
Moin Khan MD, MSc, FRCSC, Jon J. P. Warner MD
}

\section{Importance of the Topic}

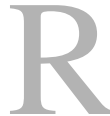

otator cuff disease accounts for more than $70 \%$ of shoulder complaints and is one of the most common musculoskeletal disorders in the adult population [11]. Among the general population, its prevalence ranges from $7 \%$ to $26 \%$ [8]. The term is broad and encompasses acute and chronic pathology affecting the shoulder joint, including rotator

\section{A Note from the Editor-In-Chief:}

We are pleased to publish the next installment of Cochrane in $\mathrm{CORR}^{\circledR}$, our partnership between $\mathrm{CORR}^{\circledR}$, The Cochrane

Collaboration ${ }^{\mathbb{R}}$, and McMaster University's Evidence-Based Orthopaedics Group. In this column, researchers from McMaster

University and other institutions will provide expert perspective on an abstract originally published in The Cochrane Library that we think is especially important.

(Page MJ, Green S, McBain B, Surace SJ, Deitch J, Lyttle N, Mrocki MA, Buchbinder R. Manual therapy and exercise for rotator cuff disease. Cochrane Database of Systematic Reviews 2016, Issue 6. Art. No.: CD012224. DOI: 10.1002/

14651858.CD012224)

Copyright (C) 2016 The Cochrane

Collaboration. Published by John Wiley \&

Sons, Ltd. Reproduced with permission.

The authors certify that neither they, nor any members of their immediate families, have cuff tears, tendonitis, and other similar pathologies. Individuals affected by this condition commonly describe debilitating pain with movement, particularly during overhead activities, as well as pain being worse at night. In the United States, rotator cuff tendinopathy accounts for approximately 4.5 million annual physician visits, with treatment and management reaching an estimated USD 3 billion annually. If we include indirect costs,

any commercial associations (such as consultancies, stock ownership, equity interest, patent/licensing arrangements, etc.) that might pose a conflict of interest in connection with the submitted article. All ICMJE Conflict of Interest Forms for authors and Clinical Orthopaedics and Related Research ${ }^{\mathbb{R}}$ editors and board members are on file with the publication and can be viewed on request.

The opinions expressed are those of the writers, and do not reflect the opinion or policy of $C O R R^{\circledR}$ or The Association of Bone and Joint Surgeons ${ }^{\mathbb{R}}$

Cochrane Reviews are regularly updated as new evidence emerges and in response to feedback, and The Cochrane Library (http:// www.thecochranelibrary.com) should be consulted for the most recent version of the review.

This Cochrane in CORR $^{\circledR}$ column refers to the abstract available at: DOI: 10.1002/

14651858.CD012224. such as lost time from work, this number is even larger [6].

Generally, initial treatment options include manual therapy including joint mobilization and manipulation, as well as specific exercise regimens and/or anti-inflammatory medications. The goal of physical therapy is to increase ROM, promote healing, strengthen periscapular musculature, and improve the stabilizing function of the rotator cuff $[4,7]$. When nonsurgical approaches are unsuccessful, surgical treatment includes rotator cuff débridement or repair and often subacromial decompression This Cochrane review of randomized control trials evaluated the efficacy of

M. Khan MD, MSc, FRCSC

Division of Orthopaedics, McMaster

University, Hamilton, ON, Canada

\section{J. J. P. Warner MD}

MGH Shoulder Service, Boston Shoulder

Institute, Harvard Medical School,

Massachusetts General Hospital, Boston, MA, USA

M. Khan MD, MSc, FRCSC ( $\square)$

Center for Evidence-Based Orthopaedics, 293 Wellington Street North, Suite 110,

Hamilton, ON L8L 8E7, Canada

e-mail: moinkhanmd@gmail.com 


\section{Cochrane in CORR}

exercise and/or manual therapy in the management of rotator cuff disease.

\section{Upon Closer Inspection}

Despite identifying more than 60 trials for inclusion, only one trial directly compared both exercise and manual therapy to placebo [2], which suggested a slight statistical improvement in function and little or no improvement in pain relief. When evaluating the results of such self-reported patient-reported outcomes from clinical trials, clinicians often focus on findings of statistical significance and reported $\mathrm{p}$ values, without consideration of effect sizes, confidence intervals, or whether the results have meaning in the clinical context $[3,10]$. Confidence intervals allow for an assessment of the variability in the magnitude and direction of effect, while judgments about clinical relevance, typically informed by a metric called the minimum clinically important difference (MCID), help clinicians to consider whether the intervention produces improvements in health that are large enough for the patient to care about.

In the case of the single trial in this review that evaluated exercise and manual therapy compared to placebo, patients who received the active treatment had an improvement in physical function greater, on average, than patients who received placebo; however, the effect size was below the MCID. Despite the statistical finding, the actual impact of the intervention was so small that patients are unlikely to perceive that exercise and manual therapy makes a noticeable improvement in their overall shoulder pain or function [2].

Although two high-quality trials [1, 2] with small sample sizes were included in the review, the remainder of the studies included in the review were at risk of potential bias, particularly with regards to blinding, where $88 \%$ of included trials were either unblinded or blinding was unclear. In addition, selective outcome reporting was a concern; $80 \%$ of trial protocols were either not registered or outcome data were incompletely reported.

The sample sizes of the included trials in this review ranged from nine to 207 patients, which is particularly problematic when trying to examine the effect of an intervention or the additive effect of an intervention with a similar control (for example, exercise and manual therapy versus exercise alone). Small sample sizes make it difficult to detect whether true differences between treatment groups exist [12]. Several trials compared interventions to placebo or sham treatments. Although, this is an ideal approach to evaluate the efficacy of an intervention, it is important to recognize that not all placebo controls are equal and some do not have a true null treatment effect [5]. For example, differing effects may be seen with the use of mobilization, manipulation, ultrasound or laser as placebo comparators. Additionally, some trials did not distinguish between acute onset of pain and functional limits after traumatic and chronic nontraumatic etiology introducing a confounding variable of acute versus chronic rotator cuff tear management. In the former case, it is generally the approach of surgeons to operative, whereas it is usually the case in the latter group to treat with physiotherapy.

\section{Take-home Messages}

This Cochrane review evaluated the efficacy of exercise or manual therapy in the setting of rotator cuff disease, and concluded that no clinical benefit exists for such interventions over placebo or other treatments. The conclusions of this review are consistent with the American Academy of Orthopaedic Surgeons Clinical Practice Guideline Summary, which was unable to recommend for or against exercise programs for patients with rotator cuff tears due to inconclusive evidence [9].

More evidence from high-quality and adequately powered trials may produce results that differ from the conclusions of this review. Further 


\section{Cochrane in CORR}

research is required to identify which patients might benefit from such treatments given the heterogeneous nature of rotator cuff disease.
Given the limitations of the available evidence, surgeon experience and patient motivation should play an important role in determining whether exercise and/or manual therapy is attempted for individuals presenting with rotator cuff disease.

\section{Appendix}

\section{Manual therapy and exercise for rotator cuff disease (Review)}

Page MJ, Green S, McBain B, Surace SJ, Deitch J, Lyttle N, Mrocki MA, Buchbinder R 


\section{Cochrane in CORR}

[Intervention Review]

\section{Manual therapy and exercise for rotator cuff disease}

Matthew J Page ${ }^{1}$, Sally Green ${ }^{2}$, Brodwen McBain ${ }^{3}$, Stephen J Surace ${ }^{4}$, Jessica Deitch ${ }^{4}$, Nicolette Lyttle ${ }^{5}$, Marshall A Mrocki ${ }^{5}$, Rachelle Buchbinder ${ }^{5}$

${ }^{1}$ School of Public Health \& Preventive Medicine, Monash University, Melbourne, Australia. ${ }^{2}$ Australasian Cochrane Centre, School of Public Health \& Preventive Medicine, Monash University, Melbourne, Australia. ${ }^{3}$ Melbourne Hand Rehab, Melbourne, Australia. ${ }^{4}$ Monash University, Melbourne, Australia. ${ }^{5}$ Monash Department of Clinical Epidemiology, Cabrini Hospital, Department of Epidemiology and Preventive Medicine, School of Public Health and Preventive Medicine, Monash University, Malvern, Australia

Contact address: Rachelle Buchbinder, Monash Department of Clinical Epidemiology, Cabrini Hospital, Department of Epidemiology and Preventive Medicine, School of Public Health and Preventive Medicine, Monash University, Malvern, 3144, Australia. rachelle.buchbinder@monash.edu.

Editorial group: Cochrane Musculoskeletal Group.

Publication status and date: New, published in Issue 6, 2016.

Review content assessed as up-to-date: 10 March 2015

Citation: Page MJ, Green S, McBain B, Surace SJ, Deitch J, Lyttle N, Mrocki MA, Buchbinder R. Manual therapy and exercise for rotator cuff disease. Cochrane Database of Systematic Reviews 2016, Issue 6. Art. No.: CD012224. DOI: 10.1002/14651858.CD012224.

Copyright $(92016$ The Cochrane Collaboration. Published by John Wiley \& Sons, Ltd.

A B S T R A C T

Background

Management of rotator cuff disease often includes manual therapy and exercise, usually delivered together as components of a physical therapy intervention. This review is one of a series of reviews that form an update of the Cochrane review, 'Physiotherapy interventions for shoulder pain'

\section{Objectives}

To synthesise available evidence regarding the benefits and harms of manual therapy and exercise, alone or in combination, for the treatment of people with rotator cuff disease.

\section{Search methods}

We searched the Cochrane Central Register of Controlled Trials (CENTRAL; 2015, Issue 3), Ovid MEDLINE (January 1966 to March 2015), Ovid EMBASE (January 1980 to March 2015), CINAHL Plus (EBSCO, January 1937 to March 2015), ClinicalTrials.gov and the WHO ICTRP clinical trials registries up to March 2015, unrestricted by language, and reviewed the reference lists of review articles and retrieved trials, to identify potentially relevant trials.

Selection criteria

We included randomised and quasi-randomised trials, including adults with rotator cuff disease, and comparing any manual therapy or exercise intervention with placebo, no intervention, a different type of manual therapy or exercise or any other intervention (e.g. glucocorticoid injection). Interventions included mobilisation, manipulation and supervised or home exercises. Trials investigating the primary or add-on effect of manual therapy and exercise were the main comparisons of interest. Main outcomes of interest were overal pain, function, pain on motion, patient-reported global assessment of treatment success, quality of life and the number of participants experiencing adverse events.

\section{Data collection and analysis}

Two review authors independently selected trials for inclusion, extracted the data, performed a risk of bias assessment and assessed the quality of the body of evidence for the main outcomes using the GRADE approach

Manual therapy and exercise for rotator cuff disease (Review)

WILEY 


\section{Cochrane in CORR}

\section{Main results}

We included 60 trials (3620 participants), although only 10 addressed the main comparisons of interest. Overall risk of bias was low in three, unclear in 14 and high in 43 trials. We were unable to perform any meta-analyses because of clinical heterogeneity or incomplete outcome reporting. One trial compared manual therapy and exercise with placebo (inactive ultrasound therapy) in 120 participants with chronic rotator cuff disease (high quality evidence). At 22 weeks, the mean change in overall pain with placebo was 17.3 points on a 100-point scale, and 24.8 points with manual therapy and exercise (adjusted mean difference (MD) 6.8 points, $95 \%$ confidence interval (CI) -0.70 to 14.30 points; absolute risk difference $7 \%, 1 \%$ fewer to $14 \%$ more). Mean change in function with placebo was 15.6 points on a 100-point scale, and 22.4 points with manual therapy and exercise (adjusted MD 7.1 points, 95\% CI 0.30 to 13.90 points; absolute risk difference $7 \%, 1 \%$ to $14 \%$ more). Fifty-seven per cent (31/54) of participants reported treatment success with manual therapy and exercise compared with $41 \%(24 / 58)$ of participants receiving placebo (risk ratio (RR) $1.39,95 \%$ CI 0.94 to 2.03; absolute risk difference $16 \%$ ( $2 \%$ fewer to $34 \%$ more). Thirty-one per cent $(17 / 55)$ of participants reported adverse events with manual therapy and exercise compared with $8 \%$ (5/61) of participants receiving placebo (RR 3.77, 95\% CI 1.49 to 9.54; absolute risk difference $23 \%$ ( $9 \%$ to $37 \%$ more). However adverse events were mild (short-term pain following treatment).

Five trials (low quality evidence) found no important differences between manual therapy and exercise compared with glucocorticoid injection with respect to overall pain, function, active shoulder abduction and quality of life from four weeks up to 12 months. However, global treatment success was more common up to 11 weeks in people receiving glucocorticoid injection (low quality evidence). One trial (low quality evidence) showed no important differences between manual therapy and exercise and arthroscopic subacromial decompression with respect to overall pain, function, active range of motion and strength at six and 12 months, or global treatment success at four to eight years. One trial (low quality evidence) found that manual therapy and exercise may not be as effective as acupuncture plus dietary counselling and Phlogenzym supplement with respect to overall pain, function, active shoulder abduction and quality life at 12 weeks. We are uncertain whether manual therapy and exercise improves function more than oral non-steroidal antiinflammatory drugs (NSAID), or whether combining manual therapy and exercise with glucocorticoid injection provides additional benefit in function over glucocorticoid injection alone, because of the very low quality evidence in these two trials.

Fifty-two trials investigated effects of manual therapy alone or exercise alone, and the evidence was mostly very low quality. There was little or no difference in patient-important outcomes between manual therapy alone and placebo, no treatment, therapeutic ultrasound and kinesiotaping, although manual therapy alone was less effective than glucocorticoid injection. Exercise alone led to less improvement in overall pain, but not function, when compared with surgical repair for rotator cuff tear. There was little or no difference in patient-important outcomes between exercise alone and placebo, radial extracorporeal shockwave treatment, glucocorticoid injection, arthroscopic subacromial decompression and functional brace. Further, manual therapy or exercise provided few or no additional benefits when combined with other physical therapy interventions, and one type of manual therapy or exercise was rarely more effective than another.

Authors' conclusions

Despite identifying 60 eligible trials, only one trial compared a combination of manual therapy and exercise reflective of common current practice to placebo. We judged it to be of high quality and found no clinically important differences between groups in any outcome. Effects of manual therapy and exercise may be similar to those of glucocorticoid injection and arthroscopic subacromial decompression, but this is based on low quality evidence. Adverse events associated with manual therapy and exercise are relatively more frequent than placebo but mild in nature. Novel combinations of manual therapy and exercise should be compared with a realistic placebo in future trials. Further trials of manual therapy alone or exercise alone for rotator cuff disease should be based upon a strong rationale and consideration of whether or not they would alter the conclusions of this review.

\section{PLAIN LANGUAGE SUMMARY}

Manual therapy and exercise for rotator cuff disease

Background

Rotator cuff disease is a common cause of shoulder pain. People with rotator cuff disease often describe their pain as being worse at night and exacerbated by movement in specific directions including overhead activity. It is often associated with loss of function and some people describe weakness.

Manual therapy and exercise for rotator cuff disease (Review)

Copyright $\Theta 2016$ The Cochrane Collaboration. Published by John Wiley \& Sons, Ltd.

WILEY 


\section{Cochrane in CORR}

Manual therapy comprises movement of the joints and other structures by a healthcare professional (e.g. physiotherapist). Exercise includes any purposeful movement of a joint, muscle contraction or prescribed activity. The aims of both treatments are to relieve pain, increase strength and joint range, and improve function.

\section{Study characteristics}

This summary of an updated Cochrane review presents what we know from research about the benefits and harms of manual therapy and exercise compared with placebo, no intervention or any other intervention in people with rotator cuff disease. After searching for all relevant studies published up to March 2015, we included 60 trials (3620 participants), however only 10 looked at manual therapy and exercise in combination. Among the included participants, $52 \%$ were women, average age was 51 years and average duration of the condition was 11 months. The average duration of manual therapy and exercise interventions was six weeks.

Key results: one trial of manual therapy and exercise compared with placebo (inactive ultrasound therapy) for 10 weeks in people with chronic rotator cuff disease

Overall pain (higher scores mean more improvement in pain reduction)

People who had manual therapy and exercise had improvements in pain that were little or no different to people who had placebo. Improvement in pain was 6.8 points more (ranging from 0.7 points less to 14.3 points more) at 22 weeks ( $7 \%$ absolute improvement).

People who had manual therapy and exercise rated their change in pain score as 24.8 points on a scale of 0 to 100 points.

People who had placebo rated their change in pain score as 17.3 points on a scale of 0 to 100 points.

Function (higher scores mean more improvement in function)

People who had manual therapy and exercise improved slightly more than people who had placebo. Improvement in function was 7.1 points more (ranging from 0.3 to 13.9 points more) at 22 weeks ( $7 \%$ absolute improvement).

People who had manual therapy and exercise rated their change in function as 22.4 points on a scale of 0 to 100 points.

People who had placebo rated their change in function as 15.6 points on a scale of 0 to 100 points.

Treatment success

16 more people out of 100 rated their treatment as successful with manual therapy and exercise compared with placebo, $16 \%$ absolute improvement (ranging from $2 \%$ less to $34 \%$ more improvement).

Fifty-seven out of 100 people reported treatment success with manual therapy and exercise.

Forty-one out of 100 people reported treatment success with placebo.

Side effects

23 more people out of 100 people had minor side effects such as temporary pain after treatment with manual therapy and exercise compared with placebo.

Thirty-one out of 100 people reported side effects with manual therapy and exercise.

Eight out of 100 people reported side effects with placebo.

Quality of the evidence

High quality evidence from one trial suggested that manual therapy and exercise improved function only slightly more than placebo at 22 weeks, was little or no different to placebo in terms of other patient-important outcomes (e.g. overall pain), and was associated with relatively more frequent but mild adverse events.

Low quality evidence suggested that there may be little or no difference in overall pain and function when manual therapy and exercise is compared with glucocorticoid injection, there may be little or no difference in overall pain and function when manual therapy and exercise is compared with arthroscopic subacromial decompression, and people who receive acupuncture plus dietary counselling and Phlogenzym supplement may have less pain and better function than people receiving manual therapy and exercise.

We are uncertain whether firstly, manual therapy and exercise improves function more than oral non-steroidal anti-inflammatory drugs (NSAID), and secondly, combining manual therapy and exercise with glucocorticoid injection provides additional improvement in function over glucocorticoid injection alone, because the quality of the evidence was very low.

Manual therapy and exercise for rotator cuff disease (Review) 


\section{Cochrane in CORR}

\section{References}

1. Beaudreuil J, Lasbleiz S, Richette P, Seguin G, Rastel C, Aout M, Vicaut E, Cohen-Solal M, Lioté F, de Vernejoul MC, Bardin T, Orcel P. Assessment of dynamic humeral centering in shoulder pain with impingement syndrome: A randomized clinical trial. Ann Rheum Dis. 2011;70:1613-1618.

2. Bennell K, Coburn S, Wee E, Green S, Harris A, Forbes A, Buchbinder R. Efficacy and cost-effectiveness of a physiotherapy program for chronic rotator cuff pathology: A protocol for a randomised, double-blind, placebo-controlled trial. $B M C$ Musculoskelet Disord. 2007;8:86.

3. Bhandari M, Montori VM, Schemitsch EH. The undue influence of significant p-values on the perceived importance of study results. Acta Orthop. 2005;76:291-295.

4. Brantingham JW, Cassa TK, Bonnefin D, Jensen M, Globe G, Hicks M, Korporaal C. Manipulative therapy for shoulder pain and disorders: Expansion of a systematic review. J Manipulative Physiol Ther. 2011;34:314-346.

5. Dowrick AS, Bhandari M. Ethical issues in the design of randomized trials: To sham or not to sham. $J$ Bone Joint Surg Am. 2012;94:7-10.

6. Judge A, Murphy RJ, Maxwell R, Arden NK, Carr AJ. Temporal trends and geographical variation in the use of subacromial decompression and rotator cuff repair of the shoulder in England. Bone Joint J. 2014;96B:70-74.

7. Littlewood C, Ashton J, ChanceLarsen K, May S, Sturrock B. Exercise for rotator cuff. Physiotherapy. 2012;98:101-109.

8. Luime JJ, Koes BW, Hendriksen IJ, Burdorf A, Verhagen AP, Miedema HS, Verhaar JA. Prevalence and incidence of shoulder pain in the general population; A systematic review. Scand $J$ Rheumatol. 2004;33:73-81.
9. Pedowitz RA, Yamaguchi K, Ahmad CS, Burks RT, Flatow EL, Green A, Iannotti JP, Miller BS, Tashjian RZ, Watters WC, Weber K, Turkelson CM, Wies JL, Anderson S, St Andre J, Boyer K, Raymond L, Sluka P, McGowan R, American Academy of Orthopaedic Surgeons. Optimizing the management of rotator cuff problems. J Am Acad Joint Surg. 2011;19:368-379.

10. Schunemann HJ, Guyatt GH. Commentary-goodbye M(C)ID! Hello MID, where do you come from? Health Serv Res. 2005;40:593-597.

11. Walker-Bone K, Palmer KT, Reading I, Coggon D, Cooper C. Prevalence and impact of musculoskeletal disorders of the upper limb in the general population. Arthritis Rheum. 2004;51:642-651.

12. Zlowodzki M, Bhandari M. Outcome measures and implications for sample-size calculations. $J$ Bone Joint Surg Am. 2009;91:35-40. 